\title{
DISTRIBUIÇÃO NATURAL DE ESPÉCIES ARBÓREAS EM ÁREAS COM DIFERENTES NÍVEIS DE ANTROPIZAÇÃO E RELAÇÃO COM OS ATRIBUTOS QUÍMICOS DO SOLO ${ }^{1}$
}

\author{
Hemerson Fernandes Calgaro ${ }^{2}$, Salatier Buzetti ${ }^{3}$, Letícia Rodrigues Silva ${ }^{4}$, Lorraine Stefanini $^{4}$, Luiz Paulo
} Montenegro de Miranda ${ }^{4}$, Marcela Aparecida de Moraes ${ }^{4}$ e Mario Luis Teixeira de Moraes ${ }^{3}$

RESUMO - Com o surgimento de áreas degradadas por ações antrópicas, os atributos químicos do solo e os caracteres silviculturais passaram a ter importância quanto ao entendimento do processo sucessional das espécies arbóreas e ao planejamento de recomposição da paisagem. Assim, este trabalho teve como objetivo avaliar a distribuição de espécies arbóreas em áreas com diferentes níveis de antropização, relacionando aspectos silviculturais com os atributos químicos do solo e caracterizando o tipo de vegetação, para fins de integração de programa de conservação genética in situ. O trabalho foi desenvolvido em área da Fazenda Experimental de Pesquisa e Extensão (FEPE) da UNESP, de Ilha Solteira, no Município de Selvíria, MS. Por meio de transecto, foram demarcadas 64 parcelas equidistantes de $50 \mathrm{~m}$ com dimensões de 10 x $10 \mathrm{~m}$, sendo 29 parcelas em área altamente antropizada (AAA), cinco em área medianamente antropizada (AMA), 15 em área pouco antropizada (APA), seis na Mata Ciliar do córrego da Véstia (Mata Ciliar) e nove na Reserva Legal. As amostras de solo foram coletadas em duas profundidades $(0,0$ a $0,20 \mathrm{~m}$ e 0,20 a $0,40 \mathrm{~m})$ para os atributos químicos, além da avaliação dos caracteres silviculturais, como altura, Diâmetro à Altura do Peito (DAP) e Forma. O estudo da distribuição natural de espécies arbóreas e condição edáfica nas diferentes áreas avaliadas permitiu concluir que os atributos químicos do solo, associados ao nível de antropização e conservação das áreas, estão influenciando a ocorrência natural, diversidade de espécies e desenvolvimento dos indivíduos arbóreos. A altura, DAP e Forma são bons indicadores para avaliar o crescimento da comunidade arbórea e relacioná-los aos atributos químicos do solo. AAPA e a Reserva Legal apresentam maior ocorrência natural, número de indivíduos e número de espécies. Na Mata Ciliar, os valores de área basal, altura, forma e atributos químicos do solo foram superiores. Das 97 espécies encontradas, seis têm potencial para serem utilizadas num programa de conservação genética in situ. São elas: Astronium fraxinifolium, Terminalia argentea, Curatella americana, Cupania vernalis, Qualea jundiahy e Andira cuyabensis.

Palavras-chave: Cerrado; Fertilidade do solo; Área degradada.

\section{NATURAL DISTRIBUTION OF TREE SPECIES IN AREAS WITH DIFFERENT LEVELS OF HUMAN DISTURBANCE AND ITS RELATION WITH CHEMICAL PROPERTIES OF THE SOIL}

\begin{abstract}
With the emergence of areas degraded by human activities, the chemical soil properties and silvicultural characters became important in understanding the succession process of tree species and planning of landscape restoration. This study aimed to evaluate the distribution of tree species in areas with different levels of human disturbance, relating silvicultural aspects to the soil chemical properties and characterizing the type of vegetation, for integration of genetic conservation program in situ. The study was conducted in the area of Research and Extension Experimental Farm (FEPE) from UNESP, Ilha Solteira, in Selvíria MS. Through transect, 64 plots were marked 50 m equidistant with dimensions of $10 \times 10 \mathrm{~m}$, where: 29 plots were in an highly disturbed area (HDA), five in moderately disturbed area (MDA), 15 in lowly disturbed
\end{abstract}

\footnotetext{
${ }^{1}$ Recebido em 18.04.2012 aceito para publicação em 15.12.2014.

${ }^{2}$ Secretaria de Agricultura e Abastecimento do Estado de São Paulo, Lins, SP - Brasil. E-mail: < hemerson.calgaro@cati.sp.gov.br>. ${ }^{3}$ Universidade Estadual Paulista "Julio de Mesquita Filho", Departamento de Fitotecnia, Tecnologia de Alimentos e SócioEconomia, Faculdade de Engenharia de Ilha Solteira-UNESP, Ilha Solteira, SP, Brasil. E-mail:<sbuzetti@agr.feis.unesp.br> e $<$ teixeira@agr.feis.unesp.br>.

${ }^{4}$ Universidade Estadual Paulista "Julio de Mesquita Filho", Programa de Pós-Graduação em Produção Vegetal, Ilha Solteira, SP - Brasil.E-mail: <luizpauloagro@yahoo.com.br>e <ma_apmoraes@yahoo.com.br>.
} 
area (LDA), six in riparian stream of Vestia river (Riparian Forest) and nine in the legal reserve. Soil samples were collected at two depths ( 0.0 to 0.20 and 0.20 to $0.40 \mathrm{~m}$ ) for the chemical analysis and the assessment of silvicultural characters, such as height, diameter at breast height (DBH) and shape. The study of the natural distribution of tree species and edaphic condition in the different evaluated areas showed that: the soil chemical properties associated with the level of human disturbance and conservation of the areas are influencing the natural occurrence, species diversity and development of the trees. Height, DBH and shape are good indicators to assess the growth of the tree community and relate them to soil chemical properties; LDA and legal reserve presented higher natural occurrence, number of individuals and number of species. In the riparian forest, basal area values, height, shape and chemical soil properties were higher. From the 97 species found, six have the potential to be used in a program of genetic conservation in situ. They are: Astronium fraxinifolium, Terminalia argentea, Curatella americana, Cupania vernalis, Qualea jundiahy and Andira cuyabensis.

Keywords: Cerrado; Soil fertility; Degraded area.

\section{INTRODUÇÃO}

Nos biomas Cerrado e Mata Atlântica, considerados hotspots brasileiros, o constante desmatamento tem ocasionado a fragmentação da vegetação natural, reduzindo o número e tamanho das populações, consequentemente associados à perda de alelos que conferem adaptação do indivíduo a um ambiente específico de colonização das espécies. A importância na reversão desse quadro se deve à maior garantia da sobrevivência dessas populações, sendo necessária a conservação ex situ de seus germoplasmas, o que garante a manutenção de genes em condições artificiais, fora do seu hábitat (PAIVA; VALOIS, 2001). Destarte, a conservação genética in situ garante a preservação do material genético justamente na área original de ocorrência, aliando condições naturais à facilidade de manejo das espécies endêmicas, auxiliando, inclusive, na recuperação de áreas degradadas.

Ações oriundas de obras de engenharia, como a utilização de horizontes superficiais para construção de aterros e barragens, resultam na retirada de solo e exposição das camadas mais profundas às condições das intempéries. Dessa forma, o restabelecimento da vegetação nativa nesses locais é dificultado, tanto pela ausência de matéria orgânica associada à baixa disponibilidade de nutrientes quanto pela presença de processos erosivos, compactação e redução da atividade biológica, conferindo baixa resiliência.

Este trabalho objetivou avaliar a distribuição de espécies arbóreas em áreas com diferentes níveis de antropização, relacionando aspectos silviculturais com os atributos químicos do solo e caracterizando o tipo de vegetação, para fins de integração do programa de conservação genética in situ.

\section{MATERIAL E MÉTODOS}

Este trabalho foi desenvolvido na Fazenda Experimental de Pesquisa e Extensão (FEPE) da UNESP - Campus de Ilha Solteira, localizada no Município de Selvíria, MS. O solo da área é classificado como Latossolo Vermelho Distrófico típico textura média, sendo a cobertura vegetal original formada por Cerrado stricto sensu que, no final da década de 1960, foi desmatado, bem como retirada uma camada de solo de 8 a $12 \mathrm{~m}$ de profundidade, servindo como área de empréstimo para a construção da barragem da Usina Hidrelétrica de Ilha Solteira. Com as alterações ocorridas na área, foi possível estabelecer divisão da paisagem considerando o grau de antropismo, por meio de transecto, por uma extensão aproximada de $3.500 \mathrm{~m}$, com parcelas de área de $100 \mathrm{~m}^{2}(10 \times 10 \mathrm{~m})$, equidistantes $50 \mathrm{~m}$. Foram demarcadas e georreferenciadas 64 parcelas ao longo do transecto, sentido norte-sul, sendo as nove parcelas iniciais marcadas na Mata de Reserva Legal (RL); com 49 parcelas atravessando a área degradada (AD), divididas pela estratificação do nível de antropização, em três áreas distintas: 29 parcelas em área altamente antropizada (AAA), cinco parcelas em área medianamente antropizada (AMA) e 15 parcelas em área pouco antropizada revegetada espontaneamente com indivíduos arbóreos em estágio avançado de regeneração (APA); e as últimas seis parcelas localizadas em Mata Ciliar (Córrego da Véstia). Utilizaram-se 6.400 $\mathrm{m}^{2}$ de área avaliada.

\subsection{Características silviculturais}

Avaliou-se a altura total da planta (ALT), em metros, utilizando aparelho hipsométrico; Diâmetro à Altura do Peito (DAP), em centímetros, obtido pela divisão do perímetro à altura do peito por $ð$. Por se tratar de

Revista Árvore, Viçosa-MG, v.39, n.2, p.233-243, 2015 
espécies nativas em condições diferentes de desenvolvimento, atribuiu-se a elas metodologia própria, adaptada e aplicada por Guerra (2008), para avaliação do fuste. Dessa maneira, estabeleceram-se notas para fuste a 2,20 m, em que a forma do tronco (FOR) variou de 1 a 5 , tanto para bifurcação (B) quanto para retidão (R), sendo a nota final dada pela média de $B+R$.

\subsection{Ocorrência, Identificação e Classificação das Espécies Arbóreas}

Todas as espécies arbóreas presentes nas 64 parcelas, desde o estágio de plântula até o estágio adulto, foram identificadas e quantificadas, bem como avaliadas aquelas de diâmetro à altura do peito igual ou superior a 0,06 m. As espécies de ocorrência natural foram classificadas, segundo Kageyama e Gandara (1993), em muito comuns (MC) (20-100 ind ha-1), comuns (C) (1-20 ind ha-1) e raras $\left(0,1-1\right.$ ind ha $\left.{ }^{-1}\right)$.

\subsection{Análise de solo}

As análises químicas do solo foram realizadas a partir de amostras, tomando como base o trabalho de Botrel et al. (2002), coletadas com trado de caneca nas profundidades de 0,0 a $0,20 \mathrm{~m}$ e 0,20 a $0,40 \mathrm{~m}$. Determinaram-se $\mathrm{P}, \mathrm{K}, \mathrm{Ca}+2$ e $\mathrm{Mg}+2$ pelo método de extração com resina trocadora de íons. A MO foi determinada por meio do método colorimétrico, o H+Al pela solução-tampão SMP e o pH por solução de $\mathrm{CaCl}_{2} 2 \mathrm{H}_{2} \mathrm{O} 0,01 \mathrm{M}$.

\subsection{Análise Estatística}

O delineamento foi em blocos casualizados desbalanceados, para a análise dos caracteres silviculturais e atributos químicos do solo e também da correlação entre área basal e atributos químicos do solo, utilizando o programa estatístico SAS (1999). O teste-F nesse caso é equivalente a um teste de comparação de médias, referindo-se à diferença entre as profundidades.

\section{RESULTADOS}

\subsection{Ocorrência de Espécies Vegetais}

Na Tabela 1 foram identificados 3.113 indivíduos, entretanto, deste total, avaliaram-se 545, por apresentarem Diâmetro à Altura do Peito (DAP) igual ou superior a 0,06 m (medida mínima comercial). Na Mata Ciliar, foram mensurados vários indivíduos das espécies Albizia polycephala, Albizia niopoides e Myracrodruon urundeuva, típicas de florestas. Em se tratando das espécies avaliadas, as áreas AAA, APA e RL foram as que mais apresentaram diversidade de espécies avaliadas, 85,89 e $70 \mathrm{sp}$, respectivamente, destacando-se a APA, que deteve a maior quantidade, mais da metade dessas plantas. As espécies não avaliadas foram apenas identificadas e quantificadas, no intuito de verificar quais estavam ocorrendo por regeneração natural e, por conseguinte, na sucessão ecológica. AAPA apresentou a maior quantidade de árvores avaliadas, destoando-se das demais áreas. Nas AAA e RL, os valores de árvores não avaliadas e total das árvores foram semelhantes.

\subsection{Caráteres Silviculturais}

Quanto à altura, as árvores na Mata Ciliar apresentaram valores médios significativamente maiores, superando todas as áreas, e a AAA exibiu o menor valor. Para o DAP, as áreas mostraram-se estatisticamente semelhantes, destacando-se a AMA e Mata Ciliar.

Este trabalho demonstrou a importância do indicador altura na avaliação de áreas em restauração de Cerrado. Na Mata Ciliar, obtiveram-se as maiores alturas e área basal, predominando algumas espécies, como Tabebuia ochracea, Myracrodruon urundeuva, A. niopoides e A. polycephala.

Quanto à Forma, os resultados indicaram que, quando os indivíduos arbóreos se encontram tutorados, eles tendem a apresentar fuste ereto. Tal fato foi observado na APA, Mata Ciliar e RL, locais com maiores valores de alturas e maiores índices populacionais. A Mata Ciliar exibiu as maiores alturas e notas da forma. Em contrapartida, na AAA e AMA, a Forma apresentou os menores valores do número de indivíduos e diversidade de espécies por parcela.

As espécies de maior área basal $(G)$ foram identificadas na Mata Ciliar $\left(29,28 \mathrm{~m}^{2} \mathrm{ha}^{-1}\right)$, AMA $\left(19,93 \mathrm{~m}^{2} \mathrm{ha}^{-1}\right)$ e RL $\left(18,17 \mathrm{~m}^{2} \mathrm{ha}^{-1}\right)$. Embora não tenha havido diferença significativa nos valores obtidos da área basal na RL e AMA, essas espécies diferiram quanto ao número de indivíduos e diversidade de espécies por parcela. Na AMA ocorreram metade da diversidade de espécies e aproximadamente metade da quantidade de indivíduos de ocorrência na RL.

Como o solo deste trabalho é o mesmo, havendo variação apenas das condições edáficas (física e química) e silviculturais, obtiveram-se valores de área basal,

Revista Árvore, Viçosa-MG, v.39, n.2, p.233-243, 2015 
Tabela 1 - Dados coletados em avaliações de campo referentes ao número de parcelas demarcadas, área em hectare, espécies e árvores avaliadas e não avaliadas, total de árvores da área de estudo e área por árvore avaliada, em área da FEPE-UNESP, no Município de Selvíria-MS.

Table 1 - Data collected in field evaluations on the number of demarcated plots, area in hectare, tree species and evaluated and not evaluated trees, total number of trees in the study area and measured area per evaluated tree in an area of FEPE-UNESP, in Selvíria-MS.

\begin{tabular}{|c|c|c|c|c|c|c|c|c|}
\hline $\begin{array}{l}\text { Áreas } \\
\text { avaliadas }\end{array}$ & $\begin{array}{c}\text { Número de parcelas } \\
\text { demarcadas }\end{array}$ & $\begin{array}{l}\text { Área } \\
\text { (ha) }\end{array}$ & $\begin{array}{l}\text { Espécies } \\
\text { avaliadas }\end{array}$ & $\begin{array}{c}\text { Espécies não } \\
\text { avaliadas }\end{array}$ & $\begin{array}{c}\text { Árvores } \\
\text { avaliadas }\end{array}$ & $\begin{array}{c}\text { Árvores } \\
\text { não avaliadas }\end{array}$ & $\begin{array}{l}\text { Total de } \\
\text { árvores }\end{array}$ & $\begin{array}{c}\mathrm{m}^{2} / \text { árvore } \\
\text { avaliada }\end{array}$ \\
\hline AAA & 29 & 0,29 & 24 & 58 & 113 & 570 & 683 & 25,70 \\
\hline AMA & 05 & 0,05 & 12 & 30 & 34 & 218 & 252 & 14,70 \\
\hline APA & 15 & 0,15 & 35 & 54 & 220 & 660 & 880 & 6,82 \\
\hline Mata ciliar & 06 & 0,06 & 15 & 17 & 58 & 707 & 765 & 10,34 \\
\hline Reserva legal & 09 & 0,09 & 28 & 42 & 120 & 413 & 533 & 7,50 \\
\hline Total & 64 & 0,64 & $64 *$ & $34 * *$ & 545 & 2.568 & 3.113 & - \\
\hline
\end{tabular}

* O total é de 64 devido ao fato de não ter somado em duplicidade as espécies encontradas em mais de uma área avaliada. Informa-se ainda que, do total de espécies identificadas (97), somente 64 foram avaliadas, em razão do fato de os indivíduos apresentarem DAP igual ou superior a $0,06 \mathrm{~m}$.

** O total é de 33 devido ao fato de não ter somado em duplicidade as espécies encontradas em mais de uma área avaliada.

tendo variado de 5,74 a 29,28 $\mathrm{m}^{2} \mathrm{ha}^{-1}$, de uma área altamente antropizada para outra preservada.

O número de indivíduos (I) na parcela foi maior na APA $(14,7)$, não apresentando diferença estatística em relação à RL $(13,1)$ e Mata ciliar $(9,7)$. Verificou-se que as áreas AAA e AMA ainda carecem de condições para expressarem estágio mais avançado de regeneração, diferentemente das demais, que exibiam condições que favoreceriam a sucessão ecológica e o desempenho das espécies. A diversidade de espécies (S) foi maior na $\operatorname{RL}(8,0)$ e na APA $(7,9)$, diferindo estatisticamente da Mata Ciliar (4,5), da AMA $(4,0)$ e da AAA $(2,5)$.

Ao todo, foram encontradas 97 espécies nos $6.400 \mathrm{~m}^{2}$, que, segundo a literatura científica, foram subdivididas em 53 espécies arbóreas oriundas do bioma Cerrado e pertencentes a 28 famílias; 34 espécies da Floresta Estacional Semidecidual (FES) e pertencentes a 15 famílias; nove espécies de transição entre Cerrado e FES, contidas em sete famílias e uma NI (não identificada) (Tabela 5). Destacaram-se, do total, seis espécies arbóreas, originárias de famílias diferentes, presentes em quatro das cinco áreas avaliadas, sendo, assim, consideradas de interesse quanto à utilização em programa de conservação genética in situ e também de recuperação de áreas degradadas. São elas: Astronium fraxinifolium, Terminalia argentea, Curatella americana, Cupania vernalis, Qualea jundiahy e Andira cuyabensis. Algumas características unem as seis espécies mais frequentes, sendo todas enquadradas como heliófitas e apenas a Qualea jundiahy como perenifólia. Entre elas, três foram pioneiras e três, secundárias. Das seis espécies, quatro foram consideradas, segundo a literatura científica, pertencentes ao bioma Cerrado, uma da FES e uma de Transição. Entre as espécies encontradas na área trabalhada, a Curatella americana, pelo seu desempenho e potencial adaptativo, pode ser considerada espécie colonizadora ou, mesmo, nucleadora (WINTERHALDER, 1996 citado por REIS, 2010), para recuperação de áreas degradadas. Essa constatação denota serem espécies com capacidade de adaptação às condições ambientais adversas. Entre essas, a Curatella americana apresentou-se sob a projeção de sua copa, em meio à serapilheira e plântulas de espécies arbóreas, em sua grande maioria frutíferas, contribuindo, sobremaneira, para a dispersão via zoocórica dos propágulos e regeneração natural da vegetação. De todas as espécies tidas como MC, 77\% encontram-se na APA, a qual apresentou o maior número de espécies e quantidade de árvores avaliadas e não avaliadas, a menor área por árvore e o maior número de indivíduos e de diversidade de espécies. Assim, essa área ofereceu condições de regeneração e nível satisfatório de resiliência. Entre as espécies encontradas nessa área, 11 (50\%) são do bioma Cerrado, nove (40\%) da Floresta Estacional Semidecidual e uma (10\%) de Transição. Destacam-se B. longifolia e M. urundeuva, classificadas como "quase ameaçadas" e "vulneráveis", respectivamente.

\subsection{Atributos Químicos do Solo}

Constataram-se no $\mathrm{P}, \mathrm{H}+\mathrm{Al}$ e Al significância na interação de área dentro de profundidade, demonstrando

Revista Árvore, Viçosa-MG, v.39, n.2, p.233-243, 2015 
estreita relação de dependência, em que as primeiras influenciam os valores silviculturais (Tabela 4). Os valores de $\mathrm{P}, \mathrm{H}+\mathrm{Al}$ e $\mathrm{Al}$ foram superiores na profundidade de 0,0 a $0,20 \mathrm{~m}$ e reduziram-se na seguinte, com destaque para o $\mathrm{P}$ na profundidade $(0,20$ a $0,40 \mathrm{~m})$, que não apresentou diferença estatística. Mesmo sendo áreas revegetadas naturalmente, $\mathrm{o} P$ comportou-se de maneira diferente; na APA, apresentou o maior valor entre as áreas degradadas e menor na RL. Entretanto, na RL obteve valor considerado de muito baixo a baixo, semelhante ao da AAA e AMA. A RL apresentou pH 3,91 , fato que propicia reação e disponibilização de íons $\mathrm{H}_{2} \mathrm{PO}_{4}$ na solução do solo; essa área ainda apresentou altos teores de $\mathrm{Al}$ e um dos maiores valores de acidez potencial e de saturação por alumínio. O teor de $\mathrm{H}+\mathrm{Al}$ foi maior na $\mathrm{RL}$, AMA e APA, na profundidade de 0,0 a 0,20 m e de 0,20 a 0,40 m. Com relação à RL, constatou-se que os teores de $\mathrm{Al}$ e os de $\mathrm{m} \%$ se relacionaram (Tabela 3 ).

Os resultados da análise de correlação dos atributos químicos do solo com a área basal nas duas profundidades de coleta $(0,0$ a $0,20 \mathrm{~m}$ e 0,20 a $0,40 \mathrm{~m})$ são apresentados na Tabela 4 . As diferenças não foram significativas para MO, CTC, $\mathrm{m} \%$ e V\% nas duas profundidades e para $\mathrm{H}+\mathrm{Al}$ na profundidade de 0,0 a $0,20 \mathrm{~m}$. Os demais resultados foram significativos e condizentes com o esperado em razão das condições encontradas nas áreas avaliadas. Destacaram-se o pH e $\mathrm{H}+\mathrm{Al}$, os quais apresentaram correlação positiva com a área basal em profundidade. O P apresentou correlação significativa na superfície amostrada, evidenciando seu caráter imóvel no solo e sua relação com o desenvolvimento vegetal.

\section{DISCUSSÃO}

\subsection{Ocorrência de Espécies Vegetais}

Nos indivíduos mensurados, houve predomínio de espécies de Cerrado, contudo a presença de espécies de Mata Atlântica caracterizou as áreas como de Transição, configurando um Ecótono (Tabela 1). Na Mata Ciliar foram descritos indivíduos, típicos de florestas, que, possivelmente, se justificam na perturbação ocorrida no passado nesse local, favorecendo o desenvolvimento dessas espécies; uma vez que a A. polycephala e A. niopoides são pioneiras. Supõe-se, ainda, que a fitofisionomia nesse local, em poucos anos, irá ser alterada pela sucessão ecológica assim que houver condições, como abertura de clareiras, uma vez que, no sub-bosque, foram encontradas espécies pioneiras e secundárias.

Em se tratando das espécies avaliadas, a AAA, APA e RL foram áreas que mais apresentaram diversidade de espécies arbóreas avaliadas. Apesar do alto grau de degradação da AAA, pode-se inferir que a vegetação está se desenvolvendo, porém, de forma lenta, em relação às demais áreas antropizadas. A RL apresentou elevado número de espécies identificadas, o que pode estar sinalizando uma condição de sucessão mais intensa.

Num estudo de ocorrência de espécies vegetais em diferentes condições de hábitat e etapas do processo sucessional na região de Assis, SP, abrangendo os ambientes de Mata Ciliar, Cerrado, vegetação em regeneração sob floresta de eucalipto, floresta secundária e área sujeita à perturbação, Saito e colaboradores (2004) encontraram nas Matas Ciliares os locais de maior diversidade de espécie, chegando até o valor de 85 sp parcela ${ }^{-1}$, valor esse muito superior ao encontrado neste estudo, na Mata Ciliar, denotando, ainda, uma possível condição de degradação. Segundo esses autores, a riqueza na diversidade de espécies tende a ser maior em ambientes ciliares, o que neste trabalho foi verificado na RL, área essa que assume também o papel de Mata Ciliar nos meses em que o rio Paraná atinge cota máxima.

Devido à ausência e presença bovina, pode estar facilitando, ou dificultando, a regeneração e sucessão entre as espécies nas áreas APA e AMA, respectivamente.

Sugere-se que o processo de regeneração e sucessão ecológica da RL está mais intenso ou não esteja ocorrendo na Mata Ciliar da mesma forma, devido à antropização e efeito de sua borda incidente.

Os valores de árvores não avaliadas e total das árvores foram semelhantes na AAA e RL, entretanto ocasionados por situações distintas, ou seja, na área primeira, provavelmente relacionado à condição de intensa degradação, imprimindo aos indivíduos arbóreos lento desenvolvimento. Na RL, esse valor deve estar associado à própria sucessão ecológica, pressupondo-se o mínimo de interferência antrópica.

Em levantamento da vegetação do Cerrado da Reserva Biológica de Moji-Guaçu, SP, Batista (1988 citado por DUBOC, 2005) encontrou variação de uma

Revista Árvore, Viçosa-MG, v.39, n.2, p.233-243, 2015 
a 16 espécies por parcela, sendo a condição edáfica o fator de maior influência na ocorrência das espécies. Corroborando esse fato, a APA e a RL apresentaram as menores áreas disponíveis para cada árvore avaliada, demonstrando intensa ocupação de indivíduos arbóreos adultos, ao contrário da AAA. Contudo, essa área, por ser a mais degradada, apresentou baixos índices de fertilidade do solo, fazendo que as espécies arbóreas não expressassem todo o potencial que, em outras áreas, é possível observar.

\subsection{Caráteres Silviculturais}

A altura dos indivíduos arbóreos da AAA provavelmente esteja relacionada às condições do local, ou seja, por estar inserida em área cuja degradação se deu de forma mais intensa. Entretanto, pode-se inferir que, apesar das características diversas das áreas e da semelhança entre os valores do DAP, houve comportamento análogo entre os indivíduos arbóreos (Tabela 2).

A altura dos indivíduos arbóreos e a forma denotam a existência de correlação positiva entre esses caracteres, quando se avaliou a Mata Ciliar, o que, nesse caso, pode evidenciar influência do ambiente no desempenho da forma.

A presença de gado na AMA está, provavelmente, dificultando a regeneração natural e proporcionando pressão de seleção a favor dos indivíduos arbóreos. Em estudo do manejo silvicultural de uma vegetação inalterada de Cerrado, no Município de Assis, SP, Durigan et al. (1993) demonstraram que a área basal se portou na faixa de $15,95 \mathrm{~m}^{2} \mathrm{ha}^{-1}$, algo pouco inferior ao encontrado neste trabalho, na área de $\operatorname{RL}\left(18,17 \mathrm{~m}^{2} \mathrm{ha}^{-1}\right)$.

Em razão de esse mesmo solo estar em condições edáficas diferentes, isso sugere que a condição dele influenciou diretamente o desenvolvimento arbóreo, haja vista que Botrel et al. (2002), estudando a influência do solo sobre as variações na estrutura arbóreo-arbustiva de uma Floresta Estacional Semidecidual em Minas Gerais, observaram que para cada tipo de solo há valores significativamente distintos de área basal.

A RL representa como era o Cerrado, entretanto podem-se observar espécies de floresta semidecídua sinalizando a ocorrência de um ecótono, também constatado por Aragão (2008). Essa área é influenciada pela elevação do nível de água do reservatório e ocorrência de fogo. As estimativas de espécies por hectare apresentam relação direta com as características da área, o que na prática pode não ser efetiva e, ainda, a sucessão ecológica entre as espécies arbóreas nessas áreas pode estar ocorrendo de maneira mais intensa, entre outros, pelo fato de haver maior diversidade de espécies. Contudo, a quantidade de espécies nas áreas mais antropizadas correspondeu, no máximo, a 50\% a menos de espécies do que em áreas onde não houve processo antrópico.

Acredita-se que nesse local, antes da construção do reservatório da hidrelétrica, as espécies de floresta semidecidual, por exigirem solos mais férteis, ocorriam como floresta ciliar e, após o avanço das águas sobre

Tabela 2 - Valores médios de altura, Diâmetro à altura do peito (DAP), Forma, área basal (G), Número de indivíduos por parcela (I) e Número de espécies por parcela (S) (sp parcela $\left.{ }^{-1}\right)$, atribuídos aos indivíduos arbóreos avaliados e presentes nas parcelas. As médias seguidas da mesma letra não diferem entre si, pelo teste de Tukey $(\mathrm{p}<0,01)$.

Table 2 - Mean values of height, Diameter at breast height (DBH), Shape, basal area (G), Number of individuals per plot (I) and Number of species per plot (S) (sp plot $\left.{ }^{-1}\right)$, assigned to individual trees evaluated and present in these plots. Means followed by the same letter do not differ by Tukey test $(p<0.01)$.

\begin{tabular}{|c|c|c|c|c|c|c|}
\hline Áreas & $\operatorname{Altura}(\mathrm{m})$ & $\mathrm{DAP}(\mathrm{cm})$ & Forma & $\mathrm{G}\left(\mathrm{m}^{2} \mathrm{ha}^{-1}\right)$ & I(unid.) & S(unid.) \\
\hline $1-\mathrm{AAA}$ & $5,62 \mathrm{c}$ & $12,42 \mathrm{bc}$ & $2,65 \mathrm{~d}$ & $5,74 \mathrm{c}$ & $3,9 \mathrm{~b}$ & $2,5 \mathrm{~b}$ \\
\hline 2- AMA & $7,78 \mathrm{~b}$ & $16,70 \mathrm{a}$ & $3,38 \mathrm{c}$ & $19,93 \mathrm{ab}$ & $7,0 \mathrm{~b}$ & $4,0 \mathrm{~b}$ \\
\hline 3- APA & $8,35 \mathrm{~b}$ & $9,73 \mathrm{c}$ & $4,15 \mathrm{ab}$ & $12,79 \mathrm{bc}$ & $14,7 \mathrm{a}$ & 7,9 a \\
\hline 4- Mata Ciliar & $11,70 \mathrm{a}$ & $14,98 \mathrm{ab}$ & 4,40 a & $29,28 \mathrm{a}$ & $9,7 \mathrm{ab}$ & $4,5 \mathrm{~b}$ \\
\hline 5- Reserva Legal (RL) & $8,62 \mathrm{~b}$ & $12,12 \mathrm{bc}$ & $4,01 \mathrm{~b}$ & $18,17 \mathrm{abc}$ & $13,1 \mathrm{a}$ & 8,0 a \\
\hline Média & 8,16 & 11,80 & 3,79 & 12,45 & 8,52 & 4,81 \\
\hline CV $(\%)$ & 32,50 & 53,80 & 22,20 & 34,85 & 22,20 & 19,88 \\
\hline Teste F & $\begin{array}{c}15,39 \\
(<, 0001)\end{array}$ & $\begin{array}{c}8,21 \\
(<, 0001)\end{array}$ & $\begin{array}{c}19,41 \\
(<, 0001)\end{array}$ & $\begin{array}{c}8,31 \\
(0,0001)\end{array}$ & $\begin{array}{c}18,64 \\
(<0,0001)\end{array}$ & $\begin{array}{c}18,87 \\
(<0,0001)\end{array}$ \\
\hline
\end{tabular}

Obs.: Os dados de média são os originais; os do teste $\mathrm{F}$ e CV foram obtidos com base na transformação raiz quadrada de $\mathrm{x}+0,5$.

Revista Árvore, Viçosa-MG, v.39, n.2, p.233-243, 2015 
esse ambiente, houve diminuição da área dessas espécies. Como consequência, as águas chegaram aos locais altos do relevo, onde havia vegetação de Cerrado, e a alta umidade desses locais proporcionou condições de desenvolvimento às espécies de floresta, iniciando o processo de colonização.

Relacionando esses resultados com os obtidos quanto ao número de indivíduos e diversidade de espécies, representando e quantificando a regeneração natural das áreas avaliadas, Durigan G. (INSTITUTO FLORESTAL DE SÃO PAULO - SECRETARIA DO MEIOAMBIENTE FLORESTAESTADUALDEASSIS, SP, 2010 - Comunicação pessoal), relatou sobre a recuperação de áreas degradadas e a condição da respectiva resiliência que, quanto maior a diversidade de espécies vegetais utilizadas no reflorestamento, melhores serão as perspectivas de sucesso. Ainda, na vegetação de Cerrado a resiliência pode ser considerada média e com regeneração natural lenta, quando a degradação, ou a perturbação, se origina do corte e retirada de terra.

As áreas APA, Mata Ciliar e RL, têm em comum altos valores de indivíduos por parcela, dossel semifechado a fechado, baixa infestação de plantas daninhas e presença considerável de serapilheira. O exposto sugere serem essas áreas fundamentais ao desenvolvimento das espécies vegetais, uma vez que os altos índices populacionais contribuem para a permanência de água no solo e, assim, para a disponibilização e absorção de nutrientes, além do armazenamento desse recurso.

As seis espécies que se destacaram possuem características comuns e, por isso, supõe-se que foram as primeiras a se regenerarem, pelo menos, nas áreas degradadas, além de serem capazes de se desenvolverem em condições de solo não ideais. Assim, ratifica-se a importância da conservação genética in situ como meio também de salvaguardar condições que propiciem a perpetuação de outras espécies.

Com vistas a preservar a complexa estrutura e dinâmica das interações entre espécies, a continuidade dos processos evolutivos que originaram e mantêm a biodiversidade, monitorar e conhecer com detalhes a estrutura populacional das espécies arbóreas contidas nas áreas avaliadas, sugere-se que sejam criados núcleos de conservação genética in situ nas cinco áreas avaliadas. Essa proposta se adequa à condição de alta diversidade de espécies, constatada neste trabalho e pelo fato de que esse tipo de conservação requer longo prazo e várias gerações. Essa modalidade de conservação não é estática e permite interações interespecíficas, sem prejudicar a evolução e coevolução. A ocorrência natural de todas as espécies encontradas nas áreas da FEPE foi atribuída, com maior frequência, a C. americana, Q. jundiahy, C. vernalis e A. cuyabensis, classificadas como espécies MC.

\subsection{Atributos Químicos do Solo}

A carência de $\mathrm{P}$ e seu caráter imóvel no solo podem ter influenciado a não diferença estatística em profundidade (Tabela 3 ). $\mathrm{O}$ teste $\mathrm{F}$ refere-se à diferença entre as profundidades, pois, como tem apenas dois tratamentos (Tratamento 1: profundidade de $0-20 \mathrm{~cm}$ e Tratamento 2: profundidade de 20 a $40 \mathrm{~cm}$ ), nesse caso passa a ser equivalente a um teste de comparação de médias. Assim, para todos os atributos químicos do solo $(\mathrm{P}, \mathrm{H}+\mathrm{A} 1, \mathrm{Al})$ houve diferença significativa entre as profundidades. Já dentro das profundidades só não houve diferença significativa entre as áreas estudadas na profundidade de 20 a $40 \mathrm{~cm}$ nos níveis de P. Os valores de $\mathrm{P}$ apresentam comportamento semelhante aos encontrados por Silva et al. (2011), que observaram maiores teores de $\mathrm{P}\left(6 \mathrm{mg} \mathrm{dm}^{-3}\right)$ e Al $\left(5 \mathrm{mmol}_{\mathrm{c}} \mathrm{dm}^{-3}\right)$ na profundidade de 0,00 a 0,20 $\mathrm{m}$, em solo sob reflorestamento ciliar avaliado após 20 anos de plantio em área de Cerrado na FEPE de Ilha Solteira (Selvíria, MS). Em contrapartida, estudos feitos por Calgaro et al. (2008) em áreas degradadas sob solo de Cerrado constataram teor inferior de $\mathrm{P}$ $\left(1 \mathrm{mg} \mathrm{dm}^{-3}\right)$ e teor superior de $\mathrm{Al}\left(9 \mathrm{mmol}_{\mathrm{c}} \mathrm{dm}^{-3}\right)$ na profundidade de 0,00 a $0,15 \mathrm{~m}$, enfatizando que a ausência de cobertura vegetal em solos degradados altera os atributos químicos do solo, diminuindo os teores de fósforo com o aumento da acidez do solo e incremento nos teores de alumínio.

A magnitude dos fluxos de nutrientes via ciclagem aumenta na fase de fechamento das copas das árvores (dossel), quando as partes inferiores começam a perder suas folhas devido à limitação de luminosidade (RAIJ et al., 1997), o que está vinculado ainda ao caráter decíduo dos indivíduos arbóreos. Sendo P um dos elementos desse processo, associado à presença significativa de serapilheira nas áreas APA e Mata Ciliar, os valores desse nutriente se apresentaram de médios a altos, conforme relato desses autores.

Revista Árvore, Viçosa-MG, v.39, n.2, p.233-243, 2015 
Tabela 3 - Desdobramentos das interações significativas dos atributos químicos do solo fósforo (P), acidez potencial ( $\mathrm{H}+\mathrm{Al})$ e alumínio (Al), em duas profundidades e em áreas avaliadas da FEPE-UNESP, no Município de Selvíria, MS.

Table 3 - Interactions significant to the soil chemical attributes: phosphorus $(P)$, potential acidity $(H+A l)$ and aluminum (Al) at two depths and evaluated in areas of FEPE-UNESP, in Selvíria-MS.

\begin{tabular}{|c|c|c|c|}
\hline Atributos & $\mathrm{P}$ & $\mathrm{H}+\mathrm{Al}$ & $\mathrm{Al}$ \\
\hline & \multicolumn{3}{|c|}{$\longrightarrow \mathrm{mmol}_{\mathrm{c}} \mathrm{dm}^{-3}$} \\
\hline Áreas & \multicolumn{3}{|c|}{ Prof. 0,0 a $0,20 \mathrm{~m}$} \\
\hline AAA & $3,14 \mathrm{ab}$ & 18,76 a & $3,03 \mathrm{ab}$ \\
\hline AMA & $3,20 \mathrm{ab}$ & $38,60 \mathrm{~b}$ & $4,00 \mathrm{~b}$ \\
\hline APA & $12,27 \mathrm{c}$ & $39,93 \mathrm{~b}$ & $5,00 \mathrm{bc}$ \\
\hline Mata Ciliar & $7,17 \mathrm{bc}$ & $21,50 \mathrm{a}$ & $1,00 \mathrm{a}$ \\
\hline Reserva Legal (RL) & $3,00 \mathrm{a}$ & $47,11 \mathrm{~b}$ & $8,22 \mathrm{c}$ \\
\hline \multicolumn{4}{|c|}{ Prof. 0,20 a $0,40 \mathrm{~m}$} \\
\hline AAA & $2,86^{\mathrm{ns}}$ & $16,93 \mathrm{a}$ & $1,97 \mathrm{a}$ \\
\hline AMA & $1,20^{\mathrm{ns}}$ & $28,20 \mathrm{bc}$ & $6,20 \mathrm{~b}$ \\
\hline APA & $2,90^{\mathrm{ns}}$ & $33,47 \mathrm{c}$ & $7,40 \mathrm{~b}$ \\
\hline Mata Ciliar & $2,00^{\mathrm{ns}}$ & $20,17 \mathrm{ab}$ & $0,83 \mathrm{a}$ \\
\hline Reserva Legal (RL) & $1,00^{\mathrm{ns}}$ & $31,80 \mathrm{c}$ & $7,56 \mathrm{~b}$ \\
\hline Média & 4,02 & 26,80 & 4,17 \\
\hline Teste F & $5,90 * *(0,0002)$ & $4,36 * *(0,0025)$ & $2,54 *(0,0432)$ \\
\hline CV $(\%)$ & 34,14 & 24,48 & 28,02 \\
\hline
\end{tabular}

Obs.: Os dados de média são os originais; os do teste F e CV para P e Al foram obtidos com base na transformação raiz quadrada de $\mathrm{x}+0,5 . * \mathrm{e} * *$ Significativos a $5 \%$ e $1 \%$ de significância, respectivamente.

Tabela 4 - Correlação dos atributos químicos do solo com a área basal (G) nas duas profundidades de coleta das amostras de solo, em áreas avaliadas da FEPE-UNESP, no Município de Selvíria, MS.

Table 4-Correlation of soil chemical properties with the basal area $(G)$ in both depths of soil sampling in assessed areas of FEPE-UNESP, in Selvíria-MS.

\begin{tabular}{|c|c|c|c|c|c|c|c|c|c|c|c|c|c|}
\hline $\begin{array}{l}\text { Atributos } \\
\text { Prof. }\end{array}$ & $\mathrm{P}$ & $\mathrm{MO}$ & $\mathrm{pH}$ & K & $\mathrm{Ca}$ & $\mathrm{Mg}$ & $\mathrm{H}+\mathrm{Al}$ & $\mathrm{Al}$ & $\mathrm{SB}$ & CTC & V & $\mathrm{m}$ & $\mathrm{S}$ \\
\hline \multirow[t]{2}{*}{$0,0-020 \mathrm{~m}$} & 0,55 & 0,09 & 0,38 & 0,50 & 0,40 & 0,70 & 0,31 & 0,49 & 0,75 & 0,08 & $-0,07$ & $-0,0318$ & 0,59063 \\
\hline & $(0,001 * *)$ & $(0,5945)$ & $(0,0317 *)$ & $(0,0034 * *)$ & $(0,0218 *)$ & $<, 0001^{* *}$ & $(0,07)$ & $(0,0042 * *)$ & $<, 0001 * *$ & $0,6316)$ & $(0,96)$ & $(0,86)$ & $(0,0004 * *)$ \\
\hline \multirow[t]{2}{*}{$0,20-0,40 \mathrm{~m}$} & 0,39 & $-0,16$ & 0,42 & 0,48 & 0,38 & 0,62 & 0,45 & 0,49 & 0,68 & 0,21 & 0,10 & $-0,22$ & 0,59063 \\
\hline & $(0,0284 *)$ & $(0,3778)$ & $(0,0181 *)$ & $\left(0,0046^{* *}\right)$ & $\left(0,0311^{*}\right)$ & $\left(0,0001^{* *}\right)$ & $\left(0,0087^{* *}\right)$ & $\left(0,0043^{* *}\right)$ & $0,68<, 0001 * *$ & $(0,2434)$ & $(0,5781)$ & $(0,2232)$ & $\left.0,0004^{* *}\right)$ \\
\hline
\end{tabular}

* Significativo a $5 \%$ de probabilidade e ** Significativo a $1 \%$ de probabilidade.

O baixo $\mathrm{pH}$ verificado na RL pode estar interferindo na dinâmica do P, uma vez que este deve estar sendo imobilizado pela formação de compostos insolúveis com Fe, $\mathrm{Al}$ e $\mathrm{Ca}$.

A constatação de que o teor de $\mathrm{H}+\mathrm{Al}$ foi maior na RL, AMA e APA conduz a uma associação do caráter ácido encontrado nessas áreas, fazendo que os sítios de troca catiônica sejam ocupados, na sua grande maioria, por íons $\mathrm{H}^{+}$e $\mathrm{Al}^{+3}$, em detrimento da fertilidade e disponibilização de bases de interesse agrícola. Quanto ao $\mathrm{Al}$, os maiores valores seguiram a mesma tendência da acidez potencial, demonstrando característica peculiar dos solos de Cerrado, que é a presença de Al associado ao caráter ácido. Conforme indicado por Raij et al. (1997), valores de Al acima de $5 \mathrm{mmol}_{\mathrm{c}} \mathrm{dm}^{-3}$ acarretam na saturação de Al em torno de $40 \%$.

Provavelmente, por localizar no ponto mais alto do relevo, a lixiviação das bases trocáveis na RL deve estar elevando a acidez e aumentando os teores de Al e $\mathrm{m} \%$.

Em estudo sobre a vegetação de Cerrado, Haridasan (2000) identificou espécies acumuladoras de Al, sendo Qualea grandiflora uma delas, encontrada neste trabalho, especificamente na AAA e RL, e ainda considerada espécie muito comum (MC), o que corrobora o fato de que nessa última foi onde se encontrou o maior teor de Al.

Revista Árvore, Viçosa-MG, v.39, n.2, p.233-243, 2015 
Tabela 5 - Relação de espécies arbóreas nativas encontradas e classificadas conforme seu bioma de origem (Cerrado - CER; Mata Atlântica: Floresta Estacional Semidecidual - FES e Vegetação de Transição - CER/FES), bem como das áreas onde foram encontradas, sendo (AAA: 1, AMA: 2, APA: 3, Mata Ciliar: 4, Reserva legal e RL: 5), em área da FEPE-UNESP, no Município de Selvíria, MS.

Table 5-Relation of native tree species found and classified according to its original biome (Cerrado- CER; Atlantic: semideciduous forest - FES and Transition Vegetation - CER/FES), as well as areas where they were found, being (HDA: 1, MDA: 2, LDA: 3, Riparian forest: 4, Legal reserve-RL: 5) in the area of FEPE-UNESP, in Selvíria, MS.

\begin{tabular}{|c|c|c|c|c|c|c|c|c|c|}
\hline N. & Nome científico & Família & corrência & Área & $\mathrm{N}$. & Nome científico & Família & Ocorrência & Área \\
\hline & \multicolumn{9}{|c|}{ CER } \\
\hline 1 & Astronium fraxinifolium & Anacardiaceae & $\mathrm{C}$ & $1,2,3,5$ & 28 & Plathymenia reticulata & Fabaceae & $\mathrm{C}$ & 2,5 \\
\hline 2 & Anacardium humile* & Anacardiaceae & $\mathrm{C}$ & 5 & 29 & Stryphnodendron adstringens & Fabaceae & $\mathrm{C}$ & 1,3 \\
\hline 3 & Annona crassiflora* & Annonaceae & $\mathrm{MC}$ & 2,3 & 30 & Acosmium dasycarpum* & Fabaceae & $\mathrm{C}$ & 2 \\
\hline 4 & Xylopia aromática & Annonaceae & $\mathrm{C}$ & 1,5 & 31 & Dipteryx alata* & Fabaceae & $\mathrm{MC}$ & 3 \\
\hline 5 & Annona pygmaea* & Annonaceae & $\mathrm{C}$ & $1,3,5$ & 32 & Machaerium hirtum & Fabaceae & MC & 3 \\
\hline 6 & Annona coriacea* & Annonaceae & $\mathrm{C}$ & 1,3 & 33 & Bowdichia virgilioides & Fabaceae & $\mathrm{C}$ & 5 \\
\hline 7 & Iiex cerasifolia & Aquifoliaceae & $\mathrm{C}$ & 5 & 34 & Mimosa claussenii $*$ & Fabaceae & $\mathrm{C}$ & $1,2,3,5$ \\
\hline 8 & Eremanthus erythropappus & Asteraceae & $\mathrm{C}$ & 1 & 35 & Andira paniculata* & Fabaceae & $\mathrm{C}$ & 1 \\
\hline 9 & Gochnatia polymorpha & Asteraceae & $\mathrm{C}$ & 1 & 36 & Byrsonima verbascifolia & Malpighiaceae & $\mathrm{C}$ & $1,2,5$ \\
\hline 10 & Tabebuia ochracea & Bignoniaceae & $\mathrm{C}$ & 3,4 & 37 & Heteropterys byrsonimifolia & Malpighiaceae & $\mathrm{C}$ & 1,3 \\
\hline 11 & Tabebuia aurea & Bignoniaceae & $\mathrm{C}$ & 1 & 38 & Eriotheca pubescens & Malvaceae & $\mathrm{C}$ & 1,5 \\
\hline 12 & Cybistax antisyphilitica* & Bignoniaceae & $\mathrm{C}$ & 1,3 & 39 & Miconia burchellii & Melastomataceae & $\mathrm{MC}$ & 5 \\
\hline 13 & Anemopaegma arvense* & Bignoniaceae & $\mathrm{C}$ & 1 & 40 & Brosimum gaudichaudii & Moraceae & $\mathrm{C}$ & 1 \\
\hline 14 & Pseudobombax longiflorum* & Bombacaceae & $\mathrm{MC}$ & 3,5 & 41 & Campomanesia sessiliflora* & Myrtaceae & $\mathrm{C}$ & 1 \\
\hline 15 & Caryocar brasiliense & Caryocaraceae & $\mathrm{C}$ & 1,3 & 42 & Roupala montana & Proteaceae & $\mathrm{C}$ & 3 \\
\hline 16 & Hirtella gracilipes* & Chrysobalanaceae & $\mathrm{C}$ & 2 & 43 & Tocoyena formosa & Rubiaceae & $\mathrm{MC}$ & 3 \\
\hline 17 & Terminalia argentea & Combretaceae & $\mathrm{C}$ & $1,2,3,5$ & 44 & Alibertia sessiis & Rubiaceae & $\mathrm{MC}$ & $2,3,5$ \\
\hline 18 & Connarus suberosus & Connaraceae & $\mathrm{C}$ & 1 & 45 & Magonia pubescens & Sapindaceae & $\mathrm{C}$ & 3 \\
\hline 19 & Rourea induta* & Connaraceae & $\mathrm{C}$ & 1,5 & 46 & Simarouba versicolor* & Simaroubaceae & $\mathrm{C}$ & 2 \\
\hline 20 & Curatella americana & Dilleniaceae & $\mathrm{C}$ & $1,2,3,5$ & 47 & Solanum lycocarpum* & Solanaceae & $\mathrm{C}$ & 1 \\
\hline 21 & Davilla elliptica* & Dilleniaceae & $\mathrm{C}$ & 1 & 48 & Solanum paniculatum* & Solanaceae & $\mathrm{C}$ & 1 \\
\hline 22 & Diospyros hispida & Ebenaceae & $\mathrm{C}$ & 1 & 49 & Styrax ferrugineus* & Styracaceae & $\mathrm{C}$ & $1,3,5$ \\
\hline 23 & Casearia sylvestris & Flacourtiaceae & $\mathrm{MC}$ & 1 & 50 & Qualea grandiflora & Vochysiaceae & $\mathrm{MC}$ & 1,5 \\
\hline 24 & Kielmeyera variabilis & Clusiaceae & $\mathrm{C}$ & 1 & 51 & Vochysia cinnamomea & Vochysiaceae & $\mathrm{C}$ & $3,4,5$ \\
\hline 25 & Dimorphandra mollis & Fabaceae & $\mathrm{C}$ & 3 & 52 & Salvertia convallariaeodora* & Vochysiaceae & $\mathrm{C}$ & 1 \\
\hline 26 & Bauhinia longifolia & Fabaceae & $\mathrm{C}$ & 3 & 53 & Qualea jundiahy & Vochysiaceae & $\mathrm{MC}$ & $1,2,3,5$ \\
\hline \multirow[t]{2}{*}{27} & Sclerolobium aureum* & Anacardiaceae & $\mathrm{C}$ & 5 & & & & & \\
\hline & \multicolumn{9}{|l|}{ - } \\
\hline 1 & Myracrodruon urundeuva & Anarcadiaceae & $\mathrm{C}$ & $1,3,4$ & 18 & Albizia niopoides & Mimosaceae & $\mathrm{C}$ & 4 \\
\hline 2 & Tapirira guianensis & Anarcadiaceae & $\mathrm{C}$ & 3,5 & 19 & Campomanesia xanthocarpa* & Myrtaceae & $\mathrm{C}$ & $1,2,3,5$ \\
\hline 3 & Annona cacans* & Annonaceae & $\mathrm{C}$ & 2 & 20 & Eugenia pyriformis* & Myrtaceae & MC & 2,3 \\
\hline 4 & Tabebuia roseo alba & Bignoniaceae & $\mathrm{C}$ & 3,4 & 21 & Eugenia uniflora & Myrtaceae & $\mathrm{C}$ & 1 \\
\hline 5 & Zeyheria tuberculosa* & Bignoniaceae & $\mathrm{MC}$ & 3 & 22 & Myrcia selloi & Myrtaceae & $\mathrm{C}$ & 3 \\
\hline 6 & Tabebuia heptaphylla & Bignoniaceae & $\mathrm{C}$ & 3 & 23 & Psidium Laurifolium* & Myrtaceae & $\mathrm{C}$ & 1 \\
\hline 7 & Cordia ecalyculata* & Boraginaceae & $\mathrm{C}$ & 3,4 & 24 & Psidium guajava* & Myrtaceae & $\mathrm{MC}$ & 1 \\
\hline 8 & Cordia trichotoma & Boraginaceae & $\mathrm{C}$ & 3 & 25 & Colubrina glandulosa & Rhamnaceae & MC & 5 \\
\hline 9 & Acacia polyphylla & Fabaceae & $\mathrm{C}$ & 1,4 & 26 & Rhamnidium elaeocarpus & Rhamnaceae & $\mathrm{C}$ & 3,4 \\
\hline 10 & Albizia polycephala & Fabaceae & $\mathrm{MC}$ & 3,4 & 27 & Genipa americana & Rubiaceae & $\mathrm{C}$ & 3 \\
\hline 11 & Anadenanthera peregrina & Fabaceae & $\mathrm{C}$ & 3,5 & 28 & Bathysa australis & Rubiaceae & $\mathrm{C}$ & 5 \\
\hline 12 & Machaerium villosum & Fabaceae & $\mathrm{C}$ & 3,4 & 29 & Zanthoxylum riedelianum & Rutaceae & $\mathrm{C}$ & 4 \\
\hline 13 & Hymenaea courbaril* & Fabaceae & $\mathrm{C}$ & 5 & 30 & Metrodorea nigra* & Rutaceae & $\mathrm{C}$ & 3,5 \\
\hline 14 & Inga edulis* & Fabaceae & $\mathrm{C}$ & 4 & 31 & Cupania vernalis & Sapindaceae & $\mathrm{C}$ & $2,3,4,5$ \\
\hline 15 & Inga $s p$ & Fabaceae & $\mathrm{MC}$ & 4 & 32 & Dilodendron bipinatum & Sapindaceae & $\mathrm{C}$ & 3 \\
\hline 16 & Casearia gossypiosperma & Flacourtiaceae & $\mathrm{MC}$ & 3 & 33 & Guazuma ulmifolia & Sterculiaceae & $\mathrm{C}$ & 1 \\
\hline 17 & Ocotea velutina & Lauraceae & $\mathrm{MC}$ & 5 & 34 & Luehea candicans & Tiliaceae & $\mathrm{MC}$ & 3 \\
\hline \multicolumn{10}{|c|}{$\mathrm{CER} / \mathrm{FES}$} \\
\hline 1 & Aspidosperma subincanum & Apocynaceae & $\mathrm{C}$ & 3,5 & 6 & Diptychandra aurantiaca & Fabaceae & $\mathrm{C}$ & 3 \\
\hline 2 & Jacaranda cuspidifolia & Bignoniaceae & $\mathrm{C}$ & 3 & 7 & Andira cuyabensis & Fabaceae & $\mathrm{C}$ & $1,2,3,5$ \\
\hline 3 & Cordia sellowiana & Boraginaceae & $\mathrm{MC}$ & 4 & 8 & Copaifera langsdorffii & Fabaceae & $\mathrm{MC}$ & 3 \\
\hline 4 & Mabea fistulifera* & Euphorbiaceae & $\mathrm{MC}$ & 1,5 & 9 & Pouteria ramiflora* & Sapotaceae & $\mathrm{C}$ & 5 \\
\hline 5 & Salacia elliptica* & Hippocrateaceae & $\mathrm{C}$ & 1,2 & & & & & \\
\hline 1 & não identificada & não identificada & $\mathrm{C}$ & 5 & & & & & \\
\hline
\end{tabular}

* Espécies não avaliadas.

* As espécies de ocorrência natural foram classificadas, segundo Kageyama e Gandara (1993), em muito comuns (MC) com 20 - 100 ind ha-1, comuns (C) com $1-20$ ind ha $^{-1}$ e raras (R) com $0,1-1$ ind ha ${ }^{-1}$.

Obs.: Classificação de famílias realizada pelo sistema APG II (SOUZA; LORENZI, 2005). 
Evidenciando caráter de preferência ou adaptação das espécies arbóreas a uma condição de solo ácido, constatou-se que os valores de $\mathrm{pH}$ e $\mathrm{H}+\mathrm{Al}$ apresentaram correlação positiva com a área basal. Esse comportamento em profundidade também foi constatado por Rizzini e Heringer (1962 citados por POGGIANI, 1974), que atribuíram às plantas do Cerrado o rápido crescimento da raiz primária em profundidade, em condição natural de solo.

A fertilidade do solo apresentou estreita relação com a condição de preservação em que as áreas se encontravam e, consequentemente, com a área basal, ou seja, os níveis de fertilidade foram maiores em locais onde a vegetação tinha maior ocupação.

Portanto, a reabilitação de áreas degradadas não consiste de ações isoladas, e sim de um conjunto de atividades que têm por objetivo recompor a paisagem que foi perturbada, sendo raras as pesquisas que procuram avaliar a qualidade do solo sob o enfoque da degradação (ALVES; SOUZA, 2008). Dessa forma, com a avaliação nutricional do solo se propõe auferir informações que subsidiarão estratégias eficazes na recuperação de áreas degradadas.

\section{CONCLUSÃO}

Os atributos químicos do solo, associados ao nível de antropização, estão influenciando a ocorrência natural, diversidade de espécies e desenvolvimento, bem como a altura, DAP e Forma, que são bons indicadores de crescimento da comunidade arbórea. AAPA e a Reserva legal apresentam maior ocorrência natural, número de indivíduos e número de espécies. Na Mata Ciliar, os valores de área basal, altura, forma e atributos químicos do solo são superiores e, das 97 espécies encontradas, seis têm potencial para serem utilizadas num programa de conservação genética in situ. São elas: Astronium fraxinifolium, Terminalia argentea, Curatella americana, Cupania vernalis, Qualea jundiahy e Andira cuyabensis.

\section{REFERÊNCIAS}

ALVES, M. C.; SOUZA, Z. M. Recuperação de área degradada por construção de hidroelétrica com adubação verde e corretivo. Revista Brasileira de Ciência do Solo, ViçosaMG, v. 32, p. 2505-2516, 2008.

ARAGÃO, S.F. Conservação genética in situ de espécies arbóreas que ocorrem na transição da floresta estacional semidecidual e o Cerrado em Selvíria - MS. 2008. 131f. Dissertação (Mestrado em Produção Vegetal) - Faculdade de Engenharia de Ilha Solteira, Universidade Estadual Paulista, Ilha Solteira, 2008.

BATISTA, E.A. Influência de fatores edáficos no cerrado da reserva biológica de Moji-Guaçu, SP. 1988. $188 \mathrm{f}$. Tese (Doutorado em Solos e Nutrição de Plantas) - Escola Superior de Agricultura Luiz de Queiroz - Universidade de São Paulo, Piracicaba, 1988.

BOTREL, R.T.; OLIVEIRA FILHO, A.T.; RODRIGUES, L.A.; CURI, N. Influência do solo e topografia sobre as variações da composição florística e estrutura da comunidade arbóreoarbustiva de uma floresta estacional semidecidual em Ingaí, MG. Revista Brasileira de Botânica, v.25, n.2, p.195-213, 2002.

CALGARO, H.F.; CASSIOLATO, A.M.R.; VALÉRIO FILHO, W.V.; FERNANDES, F.M.; MALTONI, K.L. Resíduos orgânicos como recondicionante de subsolo degradado e efeitos na atividade microbiana e fertilidade em cultivo de barbatimão. Revista Árvore, v.32, n.6, p.1069-1079, 2008.

DUBOC, E. Desenvolvimento inicial e nutrição de espécies arbóreas nativas sob fertilização, em plantios de recuperação de áreas de cerrado degradado. 2005. 173f. Tese (Doutorado em Energia na Agricultura) - Faculdade de Ciências Agronômicas, Universidade Estadual Paulista, Botucatu, 2005.

DURIGAN, G.; GARRIDO, L.M.A.G.; GARRIDO, M.A.O. Manejo silvicultural do cerrado em Assis - SP. In: CONGRESSO FLORESTAL

PANAMERICANO, 1., 1993, Curitiba. Anais... São Paulo: Sociedade Brasileira de Silvicultura/ Sociedade Brasileira de Engenheiros Florestais, 1993. v.1.p.374-77.

GUERRA, C. R. S. B. Conservação genética ex situ de populações naturais de Myracrodruon urundeuva fr. all. em sistema silvipastoril. 2008. 108 f. Tese (Doutorado) - Faculdade de Engenharia de Ilha Solteira, Universidade Estadual Paulista, Ilha Solteira, 2008.

Revista Árvore, Viçosa-MG, v.39, n.2, p.233-243, 2015 
HARIDASAN, M. Nutrição mineral de plantas nativas do cerrado. Revista Brasileira de Fisiologia Vegetal, Londrina, v. 12, n. 1, p. 54-64, 2000.

KAGEYAMA, P. Y.; GANDARA, F. B. Dinâmica de populações de espécies arbóreas para o manejo e a conservação. In: SIMPÓSIO DE ECOSSISTEMAS DA COSTA RASILEIRA, 3., 1993, São Paulo. Anais ... São Paulo: ACIESP, 1993. p. 1-9.

PAIVA, J.R.; VALOIS, A.C.C. Espécies selvagens e sua utilização no melhoramento. In: NASS, L.L.; VALOIS, A.C.C.; MELO, I.S.; VALADARESINGLIS, M.C. (Eds.). Recursos genéticos \& melhoramento - plantas. Fundação de Apoio à Pesquisa Agropecuária de Mato Grosso Fundação MT, Rondonópolis, MT. pp. 79-100. (Brasil)

POGGIANI, F. Efeito de alguns nutrientes sobre o crescimento inicial de duas espécies arbóreas do cerrado. Instituto de Pesquisas e Estudos Florestais - IPEF, n. 8, p. 3-12, 1974.

RAIJ, B.van; CANTARELLA, H.; QUAGGIO, J.A.; FURLANI, A.M.C. (Ed.). Recomendações de adubação e calagem para o Estado de São Paulo.
2. ed. Campinas: Instituto Agronômico: Fundação IAC, 1997. 285 p. (Boletim Técnico, 100).

SAITO, M.; DURIGAN, G.; BAITELLO, J.B.; FRANCO, G.A.D.C.; PASTORE, J.A.; AGUIAR, O.T.; NAKATA, H. Ocorrência de espécies vegetais em diferentes condições de habitat e etapas do processo sucessional na região de Assis, SP. In: DURIGAN, G.; BÔAS, O. V. (Org.). Pesquisas em conservação e recuperação ambiental no oeste paulista: resultados da cooperação Brasil-Japão. São Paulo: Secretaria do Meio Ambiente, 2004. 484p.

SILVA, A.M.; MORAES, M.L.T.; BUZETTI, S. Propriedades químicas de solo sob reflorestamento ciliar após 20 anos de plantio em área de cerrado. Revista Árvore, v.35, n.1, p.97-106, 2011.

STATISTICALANALYSIS SYSTEM INSTITUTE. SAS/STAT procedure guide for personal computers. 9.ed. Cary: 1999. 334 p.

WINTERHALDER, K. The restoration of industrially disturbed landscape in the Sudbury, Ontario mining and smelting region. [S.1.: s.n.], 1996. Disponível em: $<$ http:// www.udd.org/francais/forum 1996/

TexteWinterhalder.html> Acesso em: 24 jun. 2002. 